\title{
Rhinovirus as a rare cause of acute onset dilated cardiomyopathy due to myocarditis in a newborn: case report and review of the literature
}

\author{
Burcu Cebeci ${ }^{1 \oplus}$, Demet Oğuz ${ }^{1 \oplus}$, Emel Ataoğlu ${ }^{1 \oplus}$, Murat Elevli $^{2 \oplus}$, Canan $^{\circ}$ Yolcu $^{2 \oplus}$ \\ Departments of ${ }^{1}$ Neonatology and ${ }^{2}$ Pediatric Cardiology, Haseki Training and Research Hospital, İstanbul, Turkey.
}

\begin{abstract}
Background. Cardiomyopathies account for $1 \%$ of cardiac diseases that mainly originate from myocarditis in the form of dilated cardiomyopathy in the neonatal period. Viruses are the main cause of myocarditis resulting in dilated cardiomyopathy. Rhinovirus is the leading cause of viral respiratory infections though it is rarely severe.
\end{abstract}

Case. We report a 17 day old newborn with acute onset dilated cardiomyopathy due to myocarditis that developed after a viral respiratory infection caused by Rhinovirus who was admitted to the emergency ward with shock due to heart failure and recovered without any complications. This is the first case reporting the causal role of rhinovirus and myocarditis in the neonatal period.

Conclusions. A comprehensive approach is needed for the diagnosis of myocarditis in the case of unknown etiology and an extensive respiratory panel may be taken into consideration if there is a history or clinical symptoms of respiratory infection.

Key words: dilated cardiomyopathy, myocarditis, newborn, rhinovirus.

Cardiomyopathies (CMs) consist of a heterogeneous group of diseases with a wide spectrum of causes mainly affecting the myocardium resulting in cardiac dysfunction, heart failure and even death. Various classifications of $\mathrm{CMs}$ were determined based on anatomical structure, etiology, and pathophysiology. ${ }^{1} \quad$ Neonatal CMs include primarily affected myocardium with no valvular or vascular abnormalities that may be acquired or congenital and are responsible for $10 \%$ of all pediatric cardiac deaths during the childhood period. Incidence is estimated as 10:100 000 live birth. ${ }^{2}$ Neonatal CMs are mostly seen as dilated cardiomyopathy (DCM) in which myocarditis is the major cause of acquired conditions. ${ }^{3,4}$ Several microbial agents including

Burcu Cebeci

drburcucebeci@hotmail.com

Received 7th December 2020, revised 28th January 2021, 20th March 2021, 31st March 2021

accepted 19th April 2021. viruses, bacteria, parasites, and fungi may be infective pathogens for myocarditis and viruses have a majority rather than other agents. The most common virus detected in the pediatric population is Coxsackievirus B3 and other viruses like Adenovirus, Cytomegalovirus, Human immunodeficiency virus (HIV), Herpesvirus, Parvovirus B19, Influenza $A$ and B, Echoviruses, Epstein-Barr virus, and Hepatitis viruses can also be counted in the list. ${ }^{5-8}$

Rhinovirus, a member of the picornavirus family and the enterovirus genus, is the leading cause of viral respiratory infections (VRIs) including the common cold and rare but lifethreatening infections such as viral meningitis, encephalitis, myocarditis, and neonatal sepsislike syndrome. ${ }^{9}$ It is responsible for more than $\% 50$ respiratory tract infections and it causes worldwide outbreaks. Significant consequences like severe lower respiratory tract infection, myocarditis, or death may be seen in the neonatal period. ${ }^{7}$ 
Here we report a case of acute onset dilated cardiomyopathy due to myocarditis developed after VRI caused by Rhinovirus. This is the first case in the literature that reported the causal role of Rhinovirus and myocarditis in a newborn. However, myocarditis is a serious condition that may progress to heart failure, our case was diagnosed, treated effectively, and recovered well without any sequelae.

\section{Case Report}

A 17 day old male was administered to the pediatric emergency ward due to poor sucking, feeding difficulty and increased respiratory distress. He was born at 37 weeks to a 20-year old mother via vaginal delivery, weighing 2920 gr. Family history was unremarkable rather than a third-degree consanguineous marriage. His mother was a healthy woman with no history of chronic disease and no routinely used medication, who had upper respiratory tract infection symptoms such as a cough for the last five days. During postnatal adaptation period the baby had no problems and was discharged on the 2nd day of life. On physical examination, he was tachycardic, tachypneic, and dyspneic. His heart rate was 186 beats/min, respiratory rate 68 breaths/min, blood pressure 42/34 (mean $27,<3 p) \mathrm{mmHg}$, body temperature $36.6{ }^{\circ} \mathrm{C}$. He had poor perfusion and weakly palpable pulses in all extremities. His liver was $4 \mathrm{~cm}$ palpable below the costal angle. A gallop rhythm was heard on cardiac examination. There was sinus tachycardia with a heart rate of 162 beats/min on electrocardiography. The $\mathrm{P}$ wave was normal. There was a low voltage ST segment (less than $10 \mathrm{~mm}$ total amplitude in limb leads) and $\mathrm{T}$ wave suppression. PR interval was 0.12 seconds, slightly prolonged when corrected to postnatal age and heartbeat (upper limit 0.11 seconds for 160-180 beats/min below 1 month of age). Chest $\mathrm{X}$-ray revealed cardiomegaly and interstitial infiltrations (Fig. 1). On echocardiography, moderate left ventricular and atrial dilatation with mild mitral regurgitation was present. There was moderate left systolic dysfunction whereas ejection fraction (EF) was $43 \%$ by

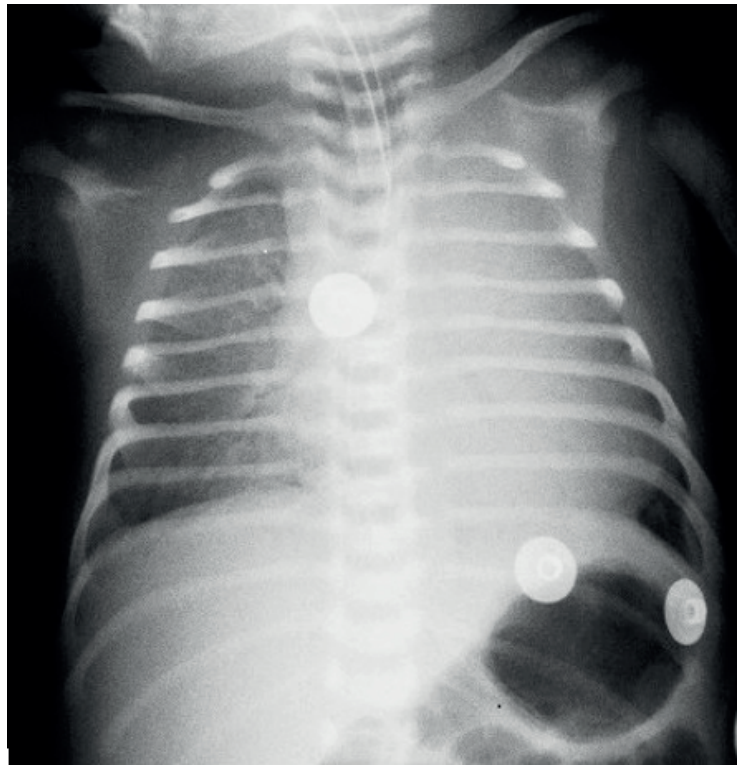

Fig. 1. Cardiomegaly and pulmonary interstitial infiltration on X-ray of the newborn.

Simpson method. One myocardial trabeculation was prominent in the left ventricular cavity. Blood gas parameters taken in the emergency unit were as follows: $\mathrm{pH}: 7.16, \mathrm{pCO}_{2}: 37$ $\mathrm{mmHg}$, bicarbonate: $12.7 \mathrm{mmol} / \mathrm{L}$, and base excess: $-13.9 \mathrm{mmol} / \mathrm{L}$. He was intubated after his oxygen saturation dropped below $85 \%$ and his blood perfusion became worse, he was then transferred to our neonatal intensive care unit. He was supported with mechanical volume guarantee ventilation. An urgent fluid bolus of $20 \mathrm{~mL} / \mathrm{kg}$ was administered because of poor peripheral perfusion and milrinone was initiated for inotropic support. White blood cells (8580 cells/L) and platelet count of 197000 per microliter were in normal range and $\mathrm{C}-$ reactive protein was negative $(0.6 \mathrm{mg} / \mathrm{L})$. On biochemical analyses, all electrolytes, renal and liver markers were in normal range except for creatinine kinase (CK): $7990 \mathrm{U} / \mathrm{L}$ (normal range $<170 \mathrm{U} / \mathrm{L}$ ), CK-MB: $302 \mathrm{ng} / \mathrm{mL}$ (normal range 0.6-6.3 ng/mL), troponin I: $0.12 \mathrm{ng} / \mathrm{mL}$ (normal range $0-0.09 \mathrm{ng} / \mathrm{mL}$ ). All metabolic screening tests including tandem mass spectrometry and urine organic acids profile were studied and no abnormal results were obtained. Blood samples were taken both for bacterial culture and serum viral markers such as the TORCH 
group viruses, Coxsackievirus $A$ and $B$, Parvovirus $B 19, \mathrm{HIV}$, hepatitis viruses (Hepatitis $B$ and $C$ ) were all negative. Thyroid hormones were in the normal range. Extended respiratory panel by PCR (Biofire Diagnostics, Salt Lake City, UT) was analyzed from the nasopharyngeal swab and Rhinovirus was positive. Given the family history of flu for the last five days, this was remarkable and significant for Rhinovirus as the etiology of myocarditis and the patient was diagnosed with acute onset dilated cardiomyopathy due to myocarditis secondary to Rhinoviral respiratory infection. He was reevaluated by a pediatric cardiologist on hospital day (HD) 2. Echocardiography revealed $\mathrm{EF}$ of $\% 49$ and minimal pericardial effusion so that furosemide was also added for the treatment of congestion. The patient weaned from the ventilator after respiratory stabilization and was extubated on HD 4. Cardiac markers gradually decreased during the follow-up (Fig. 2). All treatments for heart

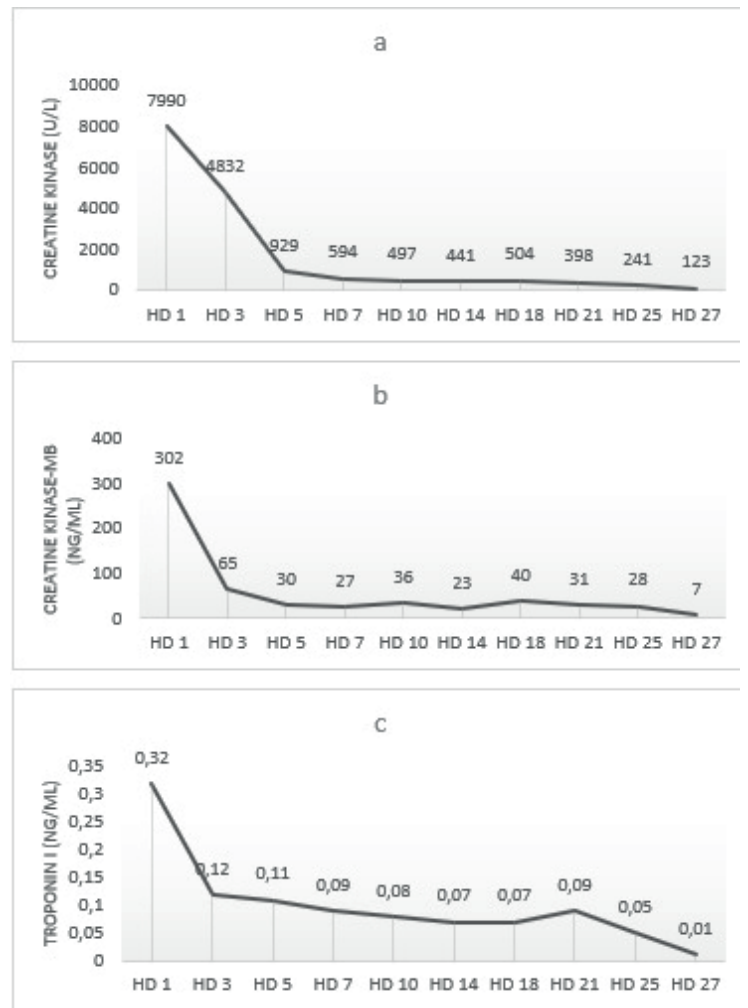

Fig. 2. Changes in cardiac enzymes of the newborn during hospitalization. failure were stopped after 3 weeks and EF improved to $\% 53$ before discharge from the hospital on HD 27. The patient recovered well without any sequelae and is still being carefully followed in our outpatient clinic, with no signs of heart failure, age-appropriate growth, and neurologic development.

Written informed consent was obtained from the parents of the patient.

\section{Discussion}

Myocarditis is a common cause of dilated cardiomyopathy among adult patients whereas it is a rarely seen cardiac inflammatory disorder having ominous consequences in the childhood period. Infants, especially newborns are more vulnerable to myocardial damage because of immature structure limiting a quick adaptive response to hemodynamic deterioration There are numerous factors (drugs, metabolic disorders, microbial agents, etc.) underlying the development of myocarditis. With more recent molecular biological techniques, more and more viruses have been identified as the cause of myocarditis. Rhinovirus is one of those mentioned as a causal agent of few cases in the childhood period in the literature. . The reason why myocarditis develops after rhinoviral infections has not been clarified yet but it's thought that both immune mediated and direct cytotoxic mechanisms of myocardium play role in the pathogenesis as it happens in all other virus borne heart diseases. ${ }^{10,11} \mathrm{~A}$ case of a 13 year old girl was presented with heart failure due to myocarditis associated with rhinoviral infection and recovered without any complication. ${ }^{12}$ Wiyatno et al reported a 4-year old boy with DCM secondary to respiratory infection and isolated Rhinovirus $C$ obtained from nasopharyngeal specimens which was known to be related to more severe illness than other species; Rhinovirus $A$ and $B .{ }^{13}$ Our main limitation is that we were unable to identify the subtype of rhinovirus with a further detailed molecular PCR method that is mainly more valuable when performed on myocardial tissue. 
The clinical presentation of myocarditis varies from mild symptoms to acute heart failure even sudden cardiac death. Unfortunately, myocarditis has a poor prognosis in the neonatal period. Mortality increases up to $75 \%$ especially in cases with Coxsackievirus $B$ whereas it is about \% 25 in the childhood population and gradually decreases. However, there is no precise data about mortality for rhinovirus as the cause of myocarditis in the literature. Our patient was presented with acute hemodynamic deterioration and had to be intubated soon after admission but was able to recover well without any complications.

Both laboratory and imaging techniques are essential diagnostic tools for myocarditis. The main cardiac biomarkers, indicating myocardial damage are creatinine kinase, $\mathrm{CK}-\mathrm{MB}$, troponin $\mathrm{I}$, and troponin $\mathrm{T}$. The level of those markers increases due to the inflammatory response involving the myocardium. Especially CK-MB and Troponin I have more diagnostic accuracy to rule out other cardiac diseases. Levels of all cardiac markers were also very high at the time of diagnosis in our cases and declined with time as a response to treatment. The echocardiogram is essential in the diagnosis and follow-up of myocarditis.

The main echocardiographic findings of our case were moderate left systolic dysfunction and only one myocardial trabeculation was prominent in the left ventricular cavity. Bilayered myocardium with prominent trabeculations seen in the left ventricular noncompaction cardiomyopathy (LVNC) is a result of an arrest in compaction during embryonic development. ${ }^{14}$ As echocardiography is the main tool to diagnose LVNC many authors focus on the size of trabeculations, the thickness, and the ratio of the compacted wall to non-compacted myocardial area. One trabeculation seen in the left ventricular cavity of our case was accepted as the consequence of DCM which disappeared after the rapid response to heart failure treatment. All medications were stopped at the 3rd week after echocardiographic findings like left systolic function and ventricular dilatation improved and clinical symptoms amended, he was discharged after 27 days of hospitalization without any complication and is still closely followed.

Endomyocardial biopsy is the gold standard for the diagnosis of myocarditis but it should be carried out by an experienced physician due to a high risk of perforation and death. The utility of endomyocardial biopsy is also limited as sampling patchy areas may lead to skipping the inflammatory involvement. We did not confirm the diagnosis of myocarditis with biopsy as our case was a newborn and the high risk of complications weighed against the benefit. To the best of our knowledge, this is the first case report showing that Rhinovirus detected as a viral agent causes myocarditis secondary to respiratory infection in a newborn. A comprehensive approach is needed for the diagnosis of myocarditis in the case of unknown etiology and an extensive respiratory panel may be taken into consideration if there is history or clinical symptoms of respiratory infection. Rapid identification of viral agents may provide prompt diagnosis, appropriate medical treatment, and prevent therapeutic challenges. Physicians may consider using an extended respiratory panel to evaluate the infectious etiology in whom other differential diagnostic diseases were excluded, especially in the neonatal period.

\section{Author contribution}

The authors confirm contribution to the paper as follows: study conception and design: $\mathrm{BC}, \mathrm{DO}$; data collection: EA, CY; analysis and interpretation of results: $\mathrm{BC}, \mathrm{ME}$, draft manuscript preparation: BC, DO. All authors reviewed the results and approved the final version of the manuscript.

\section{Conflict of interest}

The authors declare that there is no conflict of interest. 


\section{REFERENCES}

1. Elliott $\mathrm{P}$, Andersson $\mathrm{B}$, Arbustini E, et al. Classification of the cardiomyopathies: a position statement from the european society of cardiology working group on myocardial and pericardial diseases. Eur Heart J 2008; 29: 270-276. https://doi.org/10.1093/eurheartj/ ehm342

2. Bernstein D. Diseases of the myocardium. In: Kliegman RM, Stanton B, Geme JS (eds). Nelson Textbook of Pediatrics (19th ed). Philadelphia: Elsevier, 2011: 1963-1975.

3. Luk A, Ahn E, Soor GS, et al. Dilated cardiomyopathy: a review. J Clin Pathol 2009; 62: 219-225. https://doi. org/10.1136/jcp.2008.060731

4. OMIM (Online Mendelian Inheritance in Man) database. An Online Catalog of Human Genes and Genetic Disorders. Available from: https://www. omim.org

5. Krejci J, Mlejnek D, Sochorova D, Nemec P. Inflammatory cardiomyopathy: a current view on the pathophysiology, diagnosis, and treatment. Biomed Res Int 2016; 2016: 1-11. https://doi. org/10.1155/2016/4087632

6. Martin AB, Webber S, Fricker FJ, et al. Acute myocarditis. Rapid diagnosis by PCR in children. Circulation 1994; 90: 330-339. https://doi. org/10.1161/01.CIR.90.1.330

7. Howard LM, Johnson M, Gil AI, et al. Molecular epidemiology of rhinovirus detections in young children. Open Forum Infect Dis 2016; 3: ofw001. https://doi.org/10.1093/ofid/ofw001
8. Cooper LT. Myocarditis. N Engl J Med 2009; 360: 1526-1538. https://doi.org/10.1056/NEJMra0800028

9. Greenberg SB. Respiratory consequences of rhinovirus infection. Arch Intern Med 2003; 163: 278284. https://doi.org/10.1001/archinte.163.3.278

10. Kawai C. From myocarditis to cardiomyopathy: mechanisms of inflammation and cell death: learning from the past for the future. Circulation 1999; 99: 1091-1100. https://doi.org/10.1161/01.CIR.99.8.1091

11. Knowlton KU, Bardorff C. The immune system in viral myocarditis: maintaining the balance. Circ Res 1999; 85: 559-561. https://doi.org/10.1161/01. RES.85.6.559

12. Thanh DTM, Lien DM, Hanh LTH. Myocarditis related to rhinovirus. Int J Cardiol Res 2017; 4: 78-81. https://doi.org/10.19070/2470-4563-1700013

13. Wiyatno A, Febrianti ESZ, Dewantari AK, et al. Characterization of rhinovirus $C$ from a 4-year-old boy with acute onset dilated cardiomyopathy in Jakarta, Indonesia. JMM Case Rep 2018; 5: e005139. https://doi.org/10.1099/jmmcr.0.005139

14. Ross SB, Jones K, Blanch B, et al. A systematic review and meta-analysis of the prevalence of left ventricular non-compaction in adults. Eur Heart J 2020; 41: 14281436. https://doi.org/10.1093/eurheartj/ehz317 